\title{
Two-Dimensional Arrays of Self-Assembled Gold and Sulfur-Containing Fullerene Nanoparticles
}

\author{
Sheng-Ming Shih, ${ }^{\dagger}$ Wei-Fang Su,,$^{\dagger}$ Y uh-J iuan Lin, ${ }^{\ddagger}$ Cen-Shawn Wu, ${ }^{\S}$ and \\ Chii-Dong Chen $\$$
}

Institute of Materials Science and Engineering, National Taiwan University, Taipei, Taiwan, Biomedical Engineering Center, Industrial Technology Research Institute, Hsinchu, Taiwan, and Institute of Physics, Academia Sinica, Taipei, Taiwan

Recei ved November 13, 2001

\begin{abstract}
Two-dimensional (2D) arrays of gold nanoparticles with sulfur-containing fullerenenanoparticles were self-assembled through the formation of $\mathrm{Au}-\mathrm{S}$ coval ent bonds. Disulfidefunctional groups wereintroduced into the $\mathrm{C}_{60}$ molecule by reacting propyl 2-aminoethyl disulfide with $\mathrm{C}_{60}$. The $2 \mathrm{D}$ arrays were formed at the interface of the aqueous phase of gold particles and organic phase of fullerene particles as a blue transparent film. Transmission el ectronic microscope images showed that the fullerene spacing between adjacent $\mathrm{Au}(\sim 10 \mathrm{~nm})$ particles was about $2.1 \pm 0.4 \mathrm{~nm}$, which was consistent with the result of $2.18 \mathrm{~nm}$ by molecular molding calculations $\left(\mathrm{MM}^{+}\right)$. The UV-visible spectrum of this film showed a red shift and increased bandwidth due to the small spacing between gold nanoparticles. The arrays were deposited on thetop of pairs of gol d el ectrodes to form 2D coll oidal single el ectron devices. Theelectrode pairs weremade by electron beam lithographic techniques, and the separation between tips of the two el ectrodes in a pair was about $100 \mathrm{~nm}$. Electron transport measurements at low temperatures exhibited Coulomb blockade type current-voltage characteristics due to the charge effects. The assembled arrays have potential applications as nanoelectronics.
\end{abstract}

\section{Introduction}

Nanomaterials are gaining importance in the development of nanosized and high-efficient electronic devices. The chemical and physical properties of nanomaterials have been studied for over a decade because of their interesting quantum effects. When the sizes of materials reduce to nanosize scale, their optical, electric, and magnetic properties are different from those of bulk materials. ${ }^{1}$ These unusual properties are expected to be useful for the development of quantum devices such as single el ectron transistors (SETs). ${ }^{2}$

The single electron transistor is a potential candidate for the next generation of electronic devices. It possesses great advantages in low power consumption and high packing density. Since the operating temperature of a single el ectron transistor is determined solely by the geometrical size of the island(s) between the electrodes, theisland(s) should beas small as only a few nanometers. This presents a challenge to modern nanofabricaton technol ogy. Even with present day state-of-the-art electron beam lithographic technology, this is still a very demanding requi rement. On the contrary, nanostructured materials with critical dimension smaller than few nanometers can be created through the control of matter at the level of atoms and molecules, but electrical contact tothese materials is not at all trivial. Thus, self-assembly of nanostructured materials provides a potential process to fabricate high-temperature-operating nanodevices.

Many nanomaterial s have been synthesized. However, finding a method to fabricate such materials in an

\footnotetext{
* Corresponding author. E-mail: suwf@ccms.ntu.edu.tw.

+ National Taiwan University.

₹ Industrial Technology Research Institute.

$\S$ Academia Sinica.

(1) Nalwa, H. S. Handbook of Materials and Nanotechnology; Academic Press: San Diego, CA, 2000.

(2) Sato, T.; Ahmed, H.; Brown, D.; J ohnson, B. F. G. J . Appl. Phys.
} 1997, 82 (2), 696. organized fashion and on an appropriate location of circuitry effectively and efficiently is still a major challenge to scientists. There are numerous reports regarding the assembly of two-dimensional arrays of quantum dots by lithography and epitaxy depositions. ${ }^{3}$ The self-assembly methodol ogy has recently emerged as a useful technique. Many metal, insulator, and semiconductor nanoparticles havebeen assembled together with organicmolecul es such as alkyl dithiol, ${ }^{4-6}$ surfactants, $^{7-9}$ organic polymers, ${ }^{10}$ conjugated DNA, ${ }^{11,12}$ or biomimic conjugated systems. ${ }^{13}$ Two- or three-dimensional nanoparticles arrays havebeen constructed by the formation of coval ent bonds, hydrogen bonds, or van der Waals forces.

Andres et al. ${ }^{5}$ have observed an electrical conductance of a self-assembled array of gold clusters molecularly linked by aryl dithiols or aryl di-isonitriles. The selfassembled two-dimensional array of gold clusters exhi bited nonlinear Coulomb charging behavior.

In this report, novel nanoparticlearrays wereassembled and modified fullerene $\left(C_{60}\right)$ derivatives were used for assemblingnanoparticles. Weintroduced activefunctional groups (disulfide) to $\mathrm{C}_{60}$ molecules that formed twodimensional arrays with gold nanoparticles by covalent

(3) Fendler, J . H.; Meldrum, F. C. Adv. Mater. 1995, 7, 607

(4) Colvin, V. L.; Goldstein, A. N.; Alivisatos, A. P.J . Am. Chem. Soc. 1992, 114, 5221.

(5) Andres, R. P.; Bielefeld, J. D.; Henderson, J. I.; J anes, D. B.; Kolagunta, V. R.; Kubiak, C. P.; Mahoney, W.J .; Osifchin, R. G. Science 1996, 273, 1690.

(6) Cassagneau, T.; Mallouk, T. E.; Fendler, J . H.J . Am. Chem. Soc. 1998, 120, 7848

(7) Petit, C.; Taleb, A.; Pileni, M. P. Adv. Mater. 1998, 10, 259.

(8) Motte, L. Pileni, M. P. J. Phys. Chem. B 1998, 102, 4104.

(9) Li, M.; Schnablegger, H.; Mann, S. Nature 1999, 402, 393

(10) Werne, T.; Patten, T. E. J . Am. Chem. Soc. 1999, 121, 7409.

(11) Mirkin, C. A.; Letsinger, R. L.; Mucic, R. C.; Storhoff, J . . . Nature 1996, 382, 607

(12) Mucic, R. C.; Storhoff, J .J .; Mirkin, C. A.; Letsinger, R. L.J . Am. Chem. Soc. 1998, 120, 12674

(13) Connolly, S.; Fitzmaurice, D. Adv. Mater. 1999, 11, 1202. 
Scheme 1. Synthesis Scheme of (a) Fullerene Derivative $\mathbf{C}_{60}-\mathbf{1}$, (b) Fullerene Derivative $\mathbf{C}_{60}-\mathbf{2}$, and (c) Gold Nanoparticles

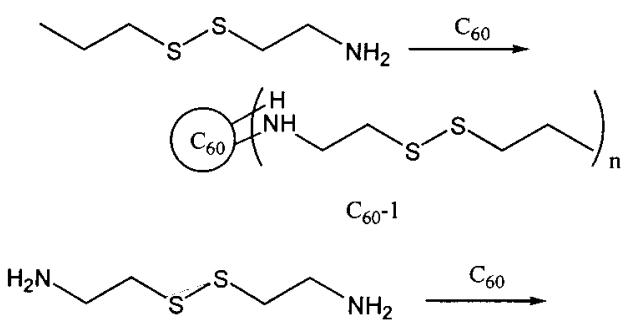

bis-2-aminoethyl disulfide<smiles>CC1(C)CCCCCC(C)(NCCSSCCNc2ccccc2)C(C)(C)N1</smiles>

$\mathrm{C}_{60^{-2}}$ ( network structure)

$$
\mathrm{HAuCl}_{4}+\text { sodium citrate } \frac{\text { reflux }}{\mathrm{H}_{2} \mathrm{O}}
$$

aqueous solution of gold nanoparticles

S-Au bonds. Then thearrays were placed on theelectrodes to fabricate single electron devices.

By combining advanced el ectron beam lithography and nanophased material assembling techniques, we have successfully prepared single electron devices with gold colloidal islands. Their electrical properties have been characterized and are presented below. In this device configuration, theself-assembled array overcamethesize limitations of lithography; thus it would provide a path for fabricating a singleelectron deviceoperableat el evated temperature.

\section{Experimental Section}

Synthesis of F ullerene Derivative $C_{60}-\mathbf{1}$. Ground $C_{60}(0.10$ g) (Aldrich Chemical ) was added to $3.4 \mathrm{~g}$ of propyl 2-aminoethyl di sulfide. The propyl 2-aminoethyl disulfide was prepared from dipropyl disulfide and 2-mercaptoethylamine according to the procedure reported by Kitsuta. ${ }^{14}$ The mixture was stirred for 3 days at room temperature until all $\mathrm{C}_{60}$ had reacted. Methanol $(20 \mathrm{~mL})$ was added into the mixture, and brown precipitates were formed. The precipitates were collected by filtration and washed with methanol several times. The product was dried with a yield of $0.096 \mathrm{~g}$ (Scheme 1a). Anal. C, 71.34; H, 4.12; N, 4.22.

Synthesis of Fullerene Derivative $\mathbf{C}_{60}-\mathbf{2}$. In a $100 \mathrm{~mL}$ round-bottomflask, $\mathrm{C}_{60}(20 \mathrm{mg})$ was dissolved in $15 \mathrm{~mL}$ of toluene. A solution $(0.63 \mathrm{~g}$ of bis-2-aminoethyl disulfide dihydrogen chloride (Aldrich Chemical ), $0.43 \mathrm{~g}$ of potassium dicarbonate, 30 $\mathrm{mL}$ of ethanol, and $10 \mathrm{~mL}$ of distilled water) was added to the $\mathrm{C}_{60}$ solution. The mixture was refluxed for $20 \mathrm{~h}$ and then cooled to room temperature. The volume of the solution was reduced to $10 \mathrm{~mL}$ using a rotor-evaporator to remove solvent. The solution was then centrifuged twice with an addition of $20 \mathrm{~mL}$ of distilled water. Brown powder ( $22 \mathrm{mg}$ ) was produced after drying under vacuum (Scheme 1b). Anal. C, 69.36; H, 3.89; N, 4.41.

Self-Assembly of Gold Nanoparticles. An aqueous solution of gold nanoparticles was synthesized by reducing tetrachloroauric acid (Across Chemical) with trisodium citrate (Across Chemical) in distilled water (Scheme 1c). Fullerene derivatives ( $\mathrm{C}_{60}-\mathbf{1}$ or $\left.\mathrm{C}_{60}-\mathbf{2}, 1 \mathrm{mg}\right)$ were dissolved $\left(\mathrm{C}_{60}-\mathbf{1}\right)$ or suspended $\left(\mathrm{C}_{60}-\mathbf{2}\right)$ in $10 \mathrm{~mL}$ of chlor oform. When a gold solution $(10 \mathrm{~mL}$ ) was poured gently into $10 \mathrm{~mL}$ of fullerene solution, a film was formed at the interface of two solutions after 2-7 days. Thefilmwas transferred onto copper grid for transmission electronic mi croscopy (TEM, J EOL 100 CX II) study. Absorption spectra were performed by

(14) Kitsuta, K. Chem. Abs. 1955, 49, 8300.

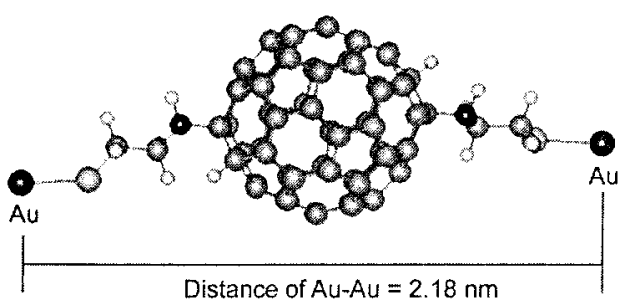

Figure 1. Molecular modeling calculations $\left(\mathrm{MM}^{+}\right)$show the $\mathrm{Au}-\mathrm{Au}$ space that is separated by a $\mathrm{C}_{60}-\mathbf{1}$ mol ecule to be 2.18 $\mathrm{nm}$. To simplify these calculations, gold atoms were used as gold nanoparticles.

directly examining the particle solutions or by transferring the films on quartz by using a UV - vis spectrometer (J ASCO V550).

Fabrication of Nanodevice. A silicon chip containing gold electrodes was dipped into the interface of gold and $\mathrm{C}_{60}-\mathbf{1}$ solutions. Thus a film of the two-dimensional array of $\mathrm{C}_{60}-\mathbf{1}$ with gold nanoparticles was transferred on the chip to linked two electrodes. After thesolvent was evaporated, thechip was washed with distilled water three times and dried by nitrogen. The el ectrodes are made by standard el ectron beam lithography and lift-off techniques. The gol d el ectrodes wereabout $50 \mathrm{~nm}$ in width and $25 \mathrm{~nm}$ in thickness, and the gap between them was about $100 \mathrm{~nm}$. The substrate was a standard Si wafer covered by a thermally grown $300 \mathrm{~nm}$ thick $\mathrm{SiO}_{2}$ layer. To reduce the capacitance of the gol d island further, the $\mathrm{SiO}_{2}$ layer surrounding the junctions was etched away prior to place assembled array. This eliminated the capacitance of the island to the ground and hel ped to increase the operating temperature.

\section{Results and Discussion}

In order for assembling gold nanoparticles by fullerene nanoparticles, we synthesized two fullerene derivatives with disulfide functional groups as shown in Scheme 1. The molecule of $\mathrm{C}_{60}$ is a buckyball of carbon, covered on its surface with unsaturated conjugated double bonds. This molecule can be attacked by more than one nucleophile. We have used two kinds of nucleophiles: (1) propyl 2-aminoethyl disulfide (Scheme 1a) and (2) bis-2-aminoethyl disulfide, which has two amino groups (Scheme 1b).

Theaminogroup attacks fullerenes and links onto their surfaces; this has been extensively studied and is well understood. ${ }^{15-18}$ Weinitial ly used 2-mercaptoethylamine to introduce thiol groups onto $\mathrm{C}_{60}$. However, the reaction of 2-mercaptoethylamine with $\mathrm{C}_{60}$ was complicated. Both the amino and thiol groups were active toward fullerene molecules. As a result, weobtained an insol uble polymeric product and that was inactive toward gold nanoparticles. Therefore, wetried to react propyl 2-aminoethyl disulfide with $\mathrm{C}_{60}$.

The disulfidegroup of this compound did not react with fullerene, and it was reactive toward gold. The reaction of propyl 2-aminoethyl disulfide with $\mathrm{C}_{60}$ proceeded as expected. Theamino group attached $\mathrm{C}_{60}$ to form $\mathrm{C}_{60}-\mathbf{1}$ and the resulting disulfide group of $\mathrm{C}_{60}-\mathbf{1}$ reacted with gold. The structure of $\mathrm{C}_{60}-\mathbf{1}$ is a ball with tentacles as shown in Figure 1. The exact positions of amino groups on the surface of $\mathrm{C}_{60}$ arenot identified. F our tentacles were found on the molecule of $\mathrm{C}_{60} \mathbf{- 1}$ from elemental analysis.

Thereaction of bis-2-aminoethyl disulfide with $\mathrm{C}_{60}$ was more complicated than that of the reaction of propyl 2-aminoethyl disulfide with $\mathrm{C}_{60}$. Both amino groups of bis-2-aminoethyl disulfide reacted with $\mathrm{C}_{60}$ and formed

(15) Seshadri, R.; Govindaraj, A.; Nagarajan, R.; Pradeep, T.; Rao, C. N. R. Tetrahedron Lett. 1992, 33, 2069.

(16) Geckeler, K. E.; Hirsch, A. J . Am. Chem. Soc. 1993, 115, 3850

(17) Kampe, K. D.; Egger, N .; Vogel, M. Angew. Chem., Int. Ed. Engl . 1993, 32, 1174

(18) Manolove, N.; Rashkov, I.; Beguin, F.; Damme, H. J . Chem. Soc., Chem. Commun. 1993, 1725. 


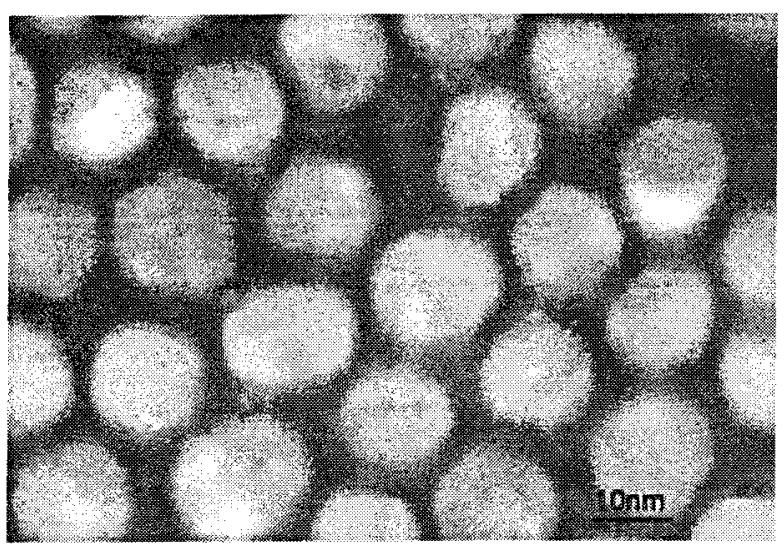

(a)

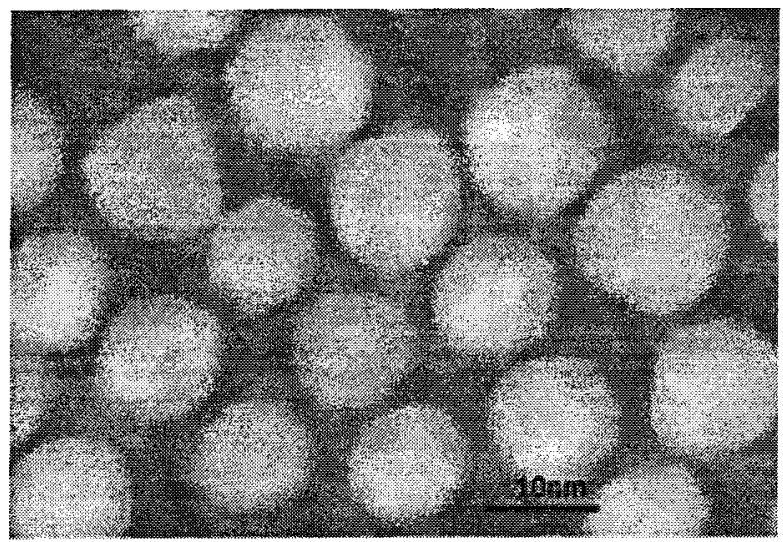

(b)

Figure 2. Micrographs of transmission electronic mi croscopy of (a) self-assembly of gol d nanoparticles and $\mathrm{C}_{60}$-1. Theaverage size of gold parti des is $14.5 \pm 2.3 \mathrm{~nm}$ and average space between gold particles is $2.1 \pm 0.4 \mathrm{~nm}$. The length of scal e bar is $10 \mathrm{~nm}$. (b) Self-assembly of gol d nanoparticles and $\mathrm{C}_{60}-\mathbf{2}$. The average size of gold partid es is $10.8 \pm 1.5 \mathrm{~nm}$ and average space between gold particles is $3.3 \pm 0.8 \mathrm{~nm}$. The length of scal e bar is $10 \mathrm{~nm}$. Somelarger gold particl es were found in both photos. They are small defects from three-dimensional arrays.

an insoluble polymeric product $\mathrm{C}_{60}-\mathbf{2}$. The exact structure of $\mathrm{C}_{60^{-}} \mathbf{2}$ has not been resolved.

The aqueous solution of gold nanoparticles was synthesized by reducing tetrachloroauricacid with tri sodium citrate in water ${ }^{19}$ (Scheme 1c). U niform size and shape of spherical gold nanoparticles was obtained. By this method, we can synthesize aqueous solution of gold nanoparticles ranging from 5 to $13 \mathrm{~nm}$.

A two-dimensional array was prepared by reacting an aqueous solution of gold nanoparticles with an organic solution of sulfur-containing fullerene nanoparticles. A thin transparent film was obtained at the interface of these two sol utions. When the disulfidegroup of fullerene derivatives reacted with gold nanoparticle, the $\mathrm{S}-\mathrm{S}$ bond brokeand two coval ent S-Au bonds formed immediately. ${ }^{20}$ We may view the arrays as superlattices that consist of gold nanoparticles and $\mathrm{C}_{60}$ nanoparticles. The gold particles were spaced by fullerene derivatives. Themolecular modeling calculations $\left(\mathrm{MM}^{+}\right)$showed that the spacing occupied by $\mathrm{C}_{60}-\mathbf{1}$ between two Au nanoparticles was 2.18 $\mathrm{nm}$ as shown in Figure 1. This distance is the sum of the

(19) Grabar, K. C.; Freeman, R. G.; Hommer, M. B.; Natan, M. J . Anal. Chem. 1995, 67, 735

(20) Nuzzo, R. G.; Zegarski, B. R.; Dubois, L. H. J . Am. Chem. Soc. 1987, 109, 733

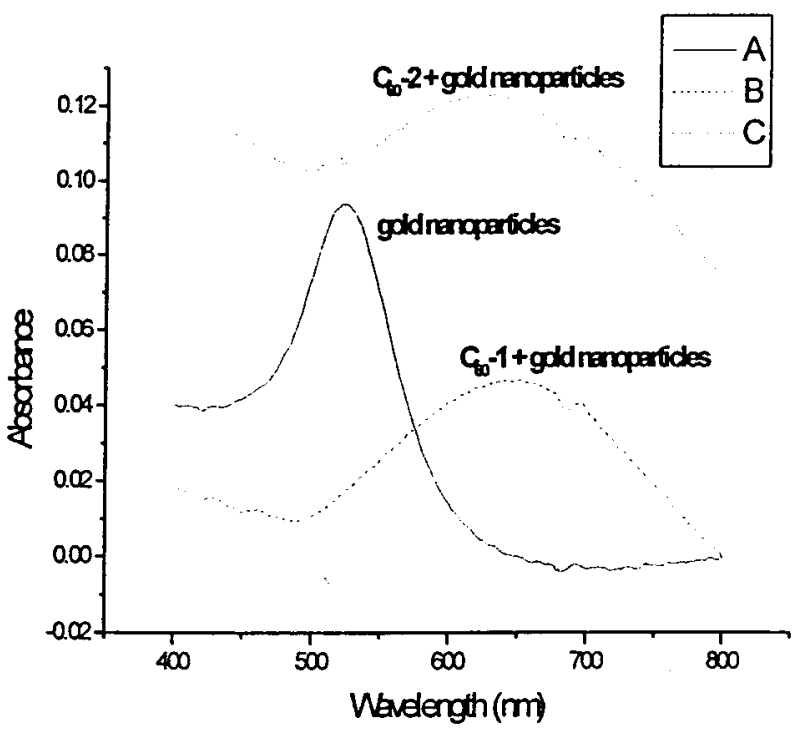

Figure 3. Ultraviol et-visiblespectra of (a) gold nanoparticles solution, line A. $\lambda_{\max }=525 \mathrm{~nm}$. (b) Nanoparticlearrays of gold and $C_{60}-1$, line B. $\lambda_{\max }=649 \mathrm{~nm}$. (c) Nanoparticle arrays of gold and $\mathrm{C}_{60}-2$, line $C$. $\lambda_{\max }=632 \mathrm{~nm}$.

diameter of one molecule of $\mathrm{C}_{60}$ and the length of two molecules of 2-mercaptoethylamine. Transmission electronic microscopy studies of the two-dimensional array made from $\mathrm{C}_{60}-\mathbf{1}$ and gol d nanoparticles indicatethat the spacing between two Au particles was an average of $2.1 \pm 0.4 \mathrm{~nm}$ (Figure 2a). This result indicated that the disulfide group of $\mathrm{C}_{60}-\mathbf{1}$ reacted with gold particles, as expected, by breaking the disulfide bond into two sulfur groups that reacted with gold particles individually.

The TEM study of $\mathrm{C}_{60}-\mathbf{2}$ assembled gold nanoparticles showed spacings with an average of $3.3 \pm 0.8 \mathrm{~nm}$ between gold particles (Figure 2b). Spacings from 2.1 to $5.1 \mathrm{~nm}$ were observed; a broad distribution was found. Also, the results show that there were more than two molecules of $\mathrm{C}_{60}$ inserted between gold particles. The fullerene derivative of $\mathrm{C}_{60}-\mathbf{2}$ is suspected to be a polymer because bis-2aminoethyl disulfide contains two active amino groups. Thegold particles havetoinsert into the polymeric matrix to form two-dimensional arrays. When the first gold particle breaks and the disulfide bond of $\mathrm{C}_{60}-\mathbf{2}$ and forms covalent bonds with it, the second gold particl efaces steric hindrance from the bulky neighboring molecule of $\mathrm{C}_{60}$. This steric hindrance effect retards the reaction of the gold particle with the second disulfide group. Consequently, disulfide groups did not react completely, especially the ones close to the reacted gold particles.

The solution of gold nanoparticles appeared dark red because of the surface plasma resonance of gold particles. The ultraviolet-visible spectrum of gold nanoparticles shows a sharp peak at $525 \mathrm{~nm}$ (visibleregion) (F igure 3a). When gold nanoparticles were assembl ed into arrays, the plasmon peak shifted toward low energy and the bandwidth increased ${ }^{21}$ (lines B and C of Figure 3). The red shift was attributed to a decreasing of spacing between gold nanoparticles. The increasing of bandwidth was due to the coagulation of gold nanoparticles. I nsomuch, a blue film was obtained when red gold nanoparticles were assembled by fullerene derivatives.

Figure 4 shows theSEM photo of two-dimensional array of gold and $\mathrm{C}_{60}-\mathbf{1}$ nanoparticlethat was transferred to the gap between two gold electrodes by the dipping method. Figure 5 shows the current-voltage characteristics of the

(21) Huang, Y.; Freiser, B. S. J . Am. Chem. Soc. 1991, 113, 9418 


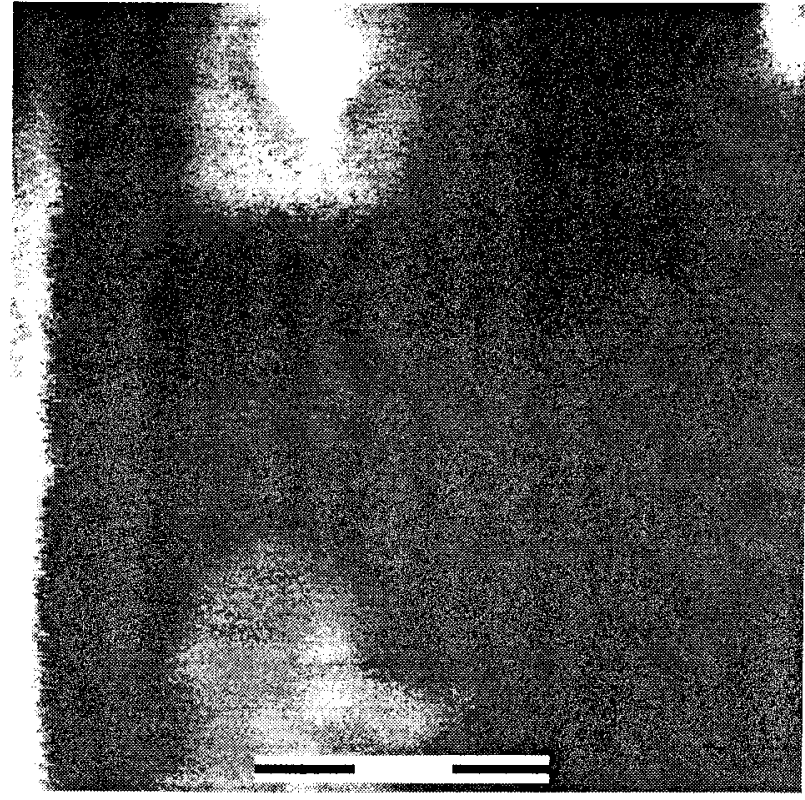

Figure 4. Micrograph of scanning electronic microscopy of two-dimensional array on the chip. This array of gol d and $\mathrm{C}_{60}-\mathbf{1}$ nanoparticle was transferred to the gap between two gold electrodes. The gold electrode was about $50 \mathrm{~nm}$ in width and $25 \mathrm{~nm}$ in thickness, and the gap between them was about 100 $\mathrm{nm}$. The length of the scale bar is $100 \mathrm{~nm}$.

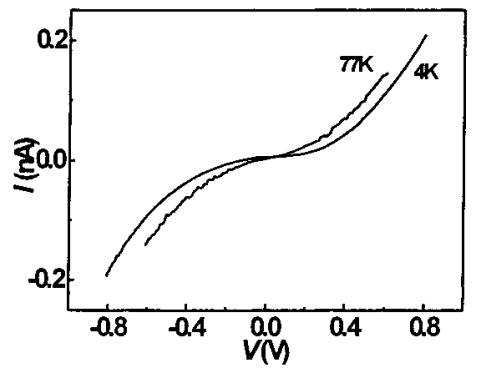

Figure 5. Current-voltage characteristics of the twodimensional array of gold and $\mathrm{C}_{60}-\mathbf{1}$ nanoparticles in the gap between two gold electrodes. The device exhibited a Coulomb gap at $4 \mathrm{~K}$.

two-dimensional array. This nonlinear behavior is more pronounced at lower temperature, which is a sign of Coulomb blockade of electron tunneling. The lower the temperature, themore Coulomb blockadewas pronounced. The device exhibited a Coulomb gap of about $0.8 \mathrm{~V}$ at 4 $K$. This device is very stable because the shape of the current-voltage characteristics was reproducible for repetitive traces of measurement at a fixed temperature.
The successful fabrication of fullerene-based twodimensional arrays offers a new class of materials with tunableelectronic properties for nanodevice applications. The $\mathrm{C}_{60}$ is electrically conducting due to the conjugating double bonds on its molecular surface. This electronic property is expected to betunable by doping different ions or atoms into the $\mathrm{C}_{60}$ buckyball or onto its surface ${ }^{22-26}$ When this phenomenon works in coordination with changing the size or el ements of particles, thereexists an enormous potential for fabrication of different type quantum devices. In our future work, we would like to fabricate a single el ectron device with a one-dimensional array of gold and $\mathrm{C}_{60}$ nanoparticles.

\section{Conclusions}

Sulfur-containing fullerene nanoparticles were synthesized by reacting amino disul fi de with $\mathrm{C}_{60}$. They were self-assembled with gold nanoparticles through the formation of Au-S covalent bonds. The 2D arrays were formed at the interface of aqueous phase of gold particles and organic phase of fullerene particles as a blue transparent film. Transmission electronic microscope images showed that the fullerene spacing between adjacent Au $(\sim 10 \mathrm{~nm})$ particles was about $2.1 \pm 0.4 \mathrm{~nm}$, which was consistent with the result of $2.18 \mathrm{~nm}$ by molecular molding calculations $\left(\mathrm{MM}^{+}\right)$. The UV-visible spectrum of this film showed a red shift and increased bandwidth due to the small spacing between gold nanoparticles. The arrays were deposited on the top of pairs of gold electrodes (spaced by $100 \mathrm{~nm}$ ) to form 2D colloidal singleel ectron devices. Electrons transport measurements at low temperatures exhibited Coulomb blockade type current-voltagecharacteristics duetothecharge effects. The assembled arrays have potential applications as nanoelectronics.

Acknowledgment. Financial support from I ndustrial Technology Research Institute (Biomedical Engineering Center) and National Science Council of the Republic of China (NSC-88-2216-E-022-041, NSC-89-2218-E-002-072, NSC-90-2218-E-002-049) are highly appreciated.

\section{$\mathrm{LA011667H}$}

(22) Roth, L. M.; Huang, Y.; Schwedler, J . T.; Cassady, C. J .; BenAmotz, D.; Kah, B.; Freiser, B. S. J Am. Chem. Soc. 1991, 113, 6298.

(23) Ohtsuki, T.; Masumoto, K.; Ohno, K.; Maruyma, Y.; Kawazoe, Y.; Sueki, K.; Kikuchi, K. Phys. Rev. Lett. 1996, 77, 3522.

(24) Szücs, Å.; Tölgyesi, M.; Csiszár, M.; Nagy, J . B.; N ovák, M. J. Electroanal. Chem. 1998, 442, 59

(25) Ohtsuki, T.; Ohno, K.; Shiga, K.; Kawazoe, Y.; Maruyma, Y.; Masumoto, K. Phys. Rev. Lett. 1998, 8, 967.

(26) Ohtsuki, T.; Ohno, K.; Shiga, K.; Kawazoe, Y.; Maruyma, Y.; Masumoto, K. J. Chem. Phys. 2000, 112, 1834. 